Bull. Mater. Sci., Vol. 39, No. 7, December 2016, pp. 1725-1733. (c) Indian Academy of Sciences.

\title{
Synthesis, characterisation and non-isothermal degradation kinetics of novel poly(mono ethylene glycol dimethacrylate-co-4-aminobenzoate)
}

\author{
A JANCIRANI ${ }^{1}$, V KOHILA $^{2}$, B MEENARATHI $^{3}$, A YELILARASI $^{4}$ and R ANBARASAN Y $^{3, *}$ (D) \\ ${ }^{1}$ Department of Chemistry, Sethu Institute of Technology, Kariapatti 626 115, India \\ ${ }^{2}$ Department of Chemistry, Kamaraj College of Engineering and Technology, Virudhunagar 626 001, India \\ ${ }^{3}$ Department of Polymer Technology, Kamaraj College of Engineering and Technology, Virudhunagar 626001 , India \\ ${ }^{4}$ Department of Physics, Kamaraj College of Engineering and Technology, Virudhunagar 626 001, India
}

MS received 29 October 2015; accepted 19 April 2016

\begin{abstract}
Synthesis of a novel co-polymer made by the addition polymerisation between MEGDMA and 4-AB by aza-Michael addition (AMA) polymerisation method is a fascinating field of research. The present investigation yielded a hazardous metal catalyst-free and toxic solvent-free methodology. The AMA polymerisation was carried out at five different $\left[M_{1} / M_{2}\right]$ values under $\mathrm{N}_{2}$ atmosphere at $100^{\circ} \mathrm{C}$ for $2 \mathrm{~h}$. Thus, obtained co-polymer was characterized by Fourier transform infrared spectroscopy, UV-visible reflectance spectroscopy, X-ray diffraction, differential scanning calorimetry, thermogravimetric analysis and scanning electron microscopy (SEM). The SEM image confirmed the formation of polymer nanoparticles. The non-isothermal degradation kinetics was followed with four different models, such as Flynn-Wall-Ozawa, Auggis-Bennet, Kissinger and Friedman method. Among the models used, the Kissinger method yielded the lowest degradation kinetics. The degradation kinetics of the co-polymer was followed with the help of model-free methods. Moreover, it was critically compared with the literature.
\end{abstract}

Keywords. Aza-Michael addition polymer; synthesis; characterisation; SEM; degradation kinetics; $E_{\mathrm{a}}$.

\section{Introduction}

The study of introduction between primary and secondary amines with carbon-carbon multiple bonds in the presence of catalyst is known as aza-Michael addition (AMA) reaction and it is a fascinating field of research now-a-days, because the methodology offers a new $\mathrm{C}-\mathrm{N}$ bond formation with excellent physical and chemical properties. In 2005, Yang et al [1] showed the ultrasound-assisted Michael addition of amines with ferrocenium under solvent-free condition at room temperature. Phenolate-catalysed aza- and thio-Michael addition in aqueous media were reported by Tamami and co-workers [2] with polymer supported Mn(II)-catalysed addition of amines with alkenes in methanol water medium, report is available in the literature [3]. Microwave-irradiated addition of amines to methyl acylates was reported in the year 2008 [4]. $\mathrm{Zn} / \mathrm{NH}_{4} \mathrm{Cl}$ protonated AMA of amines to activated alkenes was studied by Kou and research team [5]. Phosphine-catalysed Michael addition of thiol and amine with methacrylate has been reported in the literature [6]. Other authors also reported about the Michael addition reaction [7-10]. By thorough literature we could not find any report based on the Michael addition of 4-aminobenzoate (4-AB) with mono ethylene glycol dimethacrylate (MEGDMA) under catalyst and solvent-free conditions. In the present

*Author for correspondence (anbu_may3@yahoo.co.in) investigation we report this as a challenge and have successfully synthesised and characterised by various analytical techniques.

Recently, the applications of AMA reaction with diacrylate have increased due to the formation of new $\mathrm{C}-\mathrm{N}$ bond; hence, increase of molar mass leads to the formation of a typical AB-type co-polymer. Generally, the AMA reaction is accelerated with the help of a heterogeneous catalyst in the presence of a suitable solvent system [11]. In 2013, Espeel et al [12] explained the addition of the acrylate group in tetrahydrofuran medium. Mather and co-workers [13] reviewed the emerging technologies in Michael addition reaction in macromolecular design. AMA of aniline with ethyl vinyl ketone in the presence of a catalyst was reported in the literature [14]. A novel ( $\beta$-amino ester) was synthesised by Kim and research team [15]. Similarly, other Michael addition reaction with glycidyl [16], $\varepsilon$-diacrylate [17], aromatic amines [18], butane diacrylate [19], trifunctional amines [20] and aminoheptane [21] were reported. Through thorough literature survey we could not find any findings based on the Michael addition reaction on MEGDMA with 4-AB leading to the polymer formation. The novelty of the present investigation is synthesis and characterisation of 4-AB and MEGDMA-based poly(ester amine) and its non-isothermal degradation kinetics. The present investigation leads to an eco-friendly (without the use of hazardous solvents, solvent free) and economically cheaper (no catalyst addition) process. 


\section{Experimental}

\subsection{Materials}

Mono ethylene glycol dimethacrylate (MEGDMA, Merck, India), ethyl-4-aminobenzoate (4-AB, Merck, India) and sodium hydroxide pellets (Himedia, India) were purchased and used as received. Double distilled (DD) water was used for the washing purposes.

\subsection{Purification of MEGDMA}

The purification of MEGDMA was done by the distillation of $50 \mathrm{ml}$ of MEGDMA with $10 \% \mathrm{NaOH}$ solution to remove the inhibitors like quinone.

\subsection{Synthesis of poly(MEGDMA-co-4-AB)}

Five millilitres of inhibitor-free MEGDMA was polymerised with different concentrations of $4-\mathrm{AB}$ at $100^{\circ} \mathrm{C}$ for $2 \mathrm{~h}$ under $200 \mathrm{rpm}$ under magnetic stirring at $\mathrm{N}_{2}$ atmosphere [20]. At the end of the reaction yellow coloured crystals were formed. It was washed twice with distilled water to remove unreacted substances and dried for about $24 \mathrm{~h}$ inside the fuming cupboard by covering with watch glass. The polymerisation reaction is given in scheme 1 .

\subsection{Characterisation of co-polymers}

The synthesised samples were characterised by various analytical techniques, such as Fourier transform infrared (FTIR) spectroscopy, UV-visible reflectance spectroscopy, thermogravimetric analysis (TGA), differential scanning calorimetry (DSC), X-ray diffraction (XRD) study and scanning electron microscopy (SEM). FTIR spectra were recorded with the help of a Shimazdu 8400S, Japan model instrument by using the $\mathrm{KBr}$ pelletisation method from 400 to $4000 \mathrm{~cm}^{-1}$. Three milligrams of sample was ground with $200 \mathrm{mg}$ of spectral grade $\mathrm{KBr}$ and made into a disc under the pressure of seven tons. The UV-visible reflectance spectrum of the
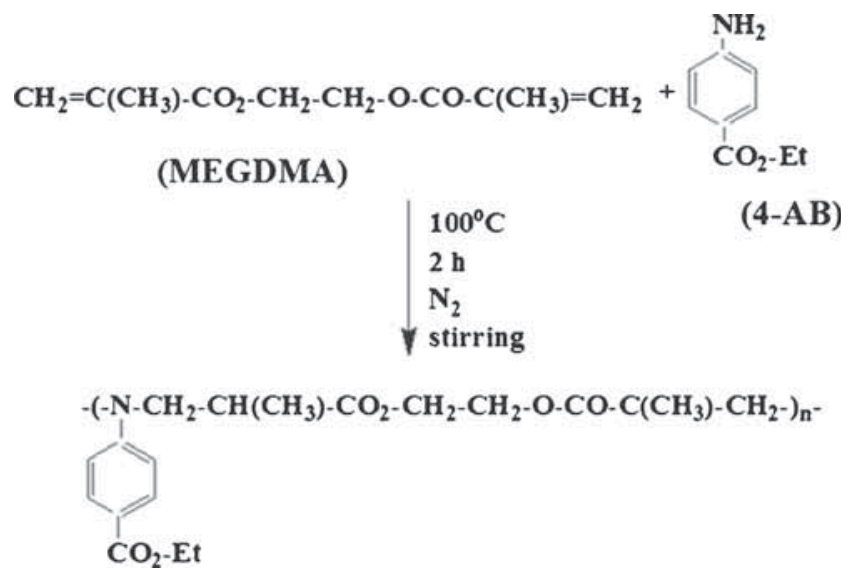

Scheme 1. Synthesis of poly(MEGDMA-co-4-AB). sample was recorded using Ocean optics USB 2G09608 instrument. XRD pattern of the samples were obtained using X'pert BROP analytical 3040 instrument. The melting temperature $\left(T_{\mathrm{m}}\right)$ of the polymer samples were determined by using DuPont Thermal Analyst 2000 DSC 910S instrument, USA. All the measurements were done under $\mathrm{N}_{2}$ atmosphere within the temperature range of room temperature to $100^{\circ} \mathrm{C}$ at the heating rate of $10^{\circ} \mathrm{C} \mathrm{min}^{-1}$. The thermal stability of polymer was measured by using a DuPont 951 thermogravimetric analyser, USA. Thermograms were recorded under air atmosphere in a temperature range of 30 to $800^{\circ} \mathrm{C}$ at different heating rates. The morphology of synthesised sample was done by SEM, JSM 6300 and JEOL, USA model instrument.

\subsection{Non-isothermal degradation kinetics}

Non-isothermal degradation kinetic studies were done by Auggis-Bennet model, Kissinger model, Flynn-Wall-Ozawa (FWO) and Friedman methods. The reaction conversion $(\alpha)$ for the degradation reaction can be determined by the following equation:

$$
\alpha=\left(\frac{W_{\mathrm{o}}-W_{\mathrm{t}}}{W_{\mathrm{o}}-W_{\mathrm{f}}}\right),
$$

where $W_{\mathrm{o}}$ is the initial weight, $W_{\mathrm{t}}$ weight at the particular temperature and $W_{\mathrm{f}}$ weight at the end of the degradation process.

2.5a FWO method: FWO [22] method is a widely used method for the study of degradation of materials under dynamic kinetic conditions. This method leads to results without making any assumptions. The equation used for this method is given below:

$$
E_{\mathrm{a}}=\frac{-R}{1.052} \times \frac{\Delta \lambda v \beta}{\Delta(1 / T)},
$$

where $E_{\mathrm{a}}$ is apparent activation energy, $R$ the gas constant, $\beta$ the heating rate and $T$ the temperature. In this method, plots of $\ln \beta$ vs. $1 / T$ produced parallel lines for each $\alpha$-value. From the slope value one can calculate the apparent activation energy.

2.5b Friedman method: Friedman [23] method is an isoconversional, model-free method, provides the following expression for thermal degradation kinetic studies based on Arrhenius equation:

$$
\ln \left\lfloor\frac{\mathrm{d} \alpha}{\mathrm{d} t}\right\rfloor=\ln (Z)+n \cdot \ln (1-\alpha)-\left\lfloor\frac{E_{\mathrm{a}}}{R_{\mathrm{t}}}\right\rfloor,
$$

where $\alpha$ is the conversion at time $t, R$ the gas constant and $T$ the temperature. The plot of $\ln (\mathrm{d} \alpha / \mathrm{d} t) v s .1 / T$ produced a linear line with the slope value of $E_{\mathrm{a}} / R$. The plots give the activation energies at different stages of degradation reaction that takes place. 
2.5c Auggis-Bennet model: This method is also a classical kinetic model. According to the method suggested by Auggis and Bennett [20],

$$
\ln \left(\frac{\beta}{T_{\mathrm{d}}}\right)=-\frac{E}{R T_{\mathrm{d}}}+\ln A,
$$

where $T_{\mathrm{d}}$ is the degradation temperature exactly determined from the differential thermal analysis curve. The activation energy $E$ can be obtained from the slope of the straight line $\ln \beta /\left(T_{\mathrm{d}}\right)$ vs. $1 / T_{\mathrm{d}}$.

2.5d Kissinger model: This is also a well-known classical kinetic analysis method. The assumption made here is that the reaction rate reaches its maximum at the temperature $\left(T_{\mathrm{d}}\right)$ at the derivative thermogravimetry peak. This assumption also gives a constant degree of conversion $(\alpha)$ at $T_{\mathrm{d}}$. In many cases, the degree of conversion $(\alpha)$ at $T_{\mathrm{d}}$ varies with the heating rate. The Kissinger equation [21] is given below:

$$
\ln \left(\frac{\beta}{T_{\mathrm{d}}^{2}}\right)=\ln \left(\frac{A R}{E}\right)-\frac{E}{R T_{\mathrm{d}}} .
$$

The activation energy $E$ can be obtained from the slope of the plot $\ln \left(\beta / T_{\mathrm{d}}^{2}\right)$ vs. $1 / T_{\mathrm{d}}$. This method provides the value of $A$ apart from the value of activation energy $E$.

2.5e Model-free methods: The model-free methods are very useful for the accurate calculation of the energy of activation $\left(E_{\mathrm{a}}\right)$. For this purpose isoconversional methods particularly differential isoconversional and integral isoconversional methods are used. The $E_{\mathrm{a}}$ value is calculated from the following differential isoconversional equation. According to the isoconversional principle, the reaction rate at constant reaction conversion $(\alpha)$ is only a function of temperature.
Thus, the logarithmic derivative for the reaction rate equation, $\mathrm{d} \alpha / \mathrm{d} t=k(T) f(\alpha)$, at constant $\alpha$ is given below,

$$
[\partial \ln (\mathrm{d} \alpha / \mathrm{d} t) / \partial(1 / T)]_{\alpha}=-\left(E_{\mathrm{a}}\right)_{\alpha} / R
$$

From equation (6), the temperature dependence of the isoconversional rate can be used to evaluate isoconversional values of the activation energy, $\left(E_{\mathrm{a}}\right)_{\alpha}$, without determining any particular form of the reaction model. Hence, the isoconversional method is also known as model-free methods [24]. In the present investigation, the researchers followed the Goswami et al study [25].

The activation energy can also be determined by an integral form of the isoconversional methods such as KissingerAkahira-Sunose (KAS) method. Compared to FWO method, the KAS method offers significantly more accurate $E_{\text {a }}$ value [26]. The KAS equation is as follows:

$$
\ln \left(\frac{\beta}{T_{\alpha}^{2}}\right)=\text { Const }-\frac{\left(E_{\mathrm{a}}\right)_{\alpha}}{R T_{\alpha}} .
$$

\section{Results and discussion}

\subsection{FTIR characterisation}

The FTIR spectrum was used to identify the functional groups present in the co-polymer. The co-polymerisation was carried out at different concentrations of $4-\mathrm{AB}$ at constant MEGDMA concentration. The other experimental conditions such as temperature, time and stirring speed were kept as constant. Figure 1a-e indicates the FTIR spectra of copolymer at different concentrations of $4-\mathrm{AB}$. The important peaks are characterised below. Peaks at 3428 and $3347 \mathrm{~cm}^{-1}$ indicate the presence of primary amine observed from 4-AB. A doublet peak around $3000 \mathrm{~cm}^{-1}$ is due to the $\mathrm{C}-\mathrm{H}$ stretching
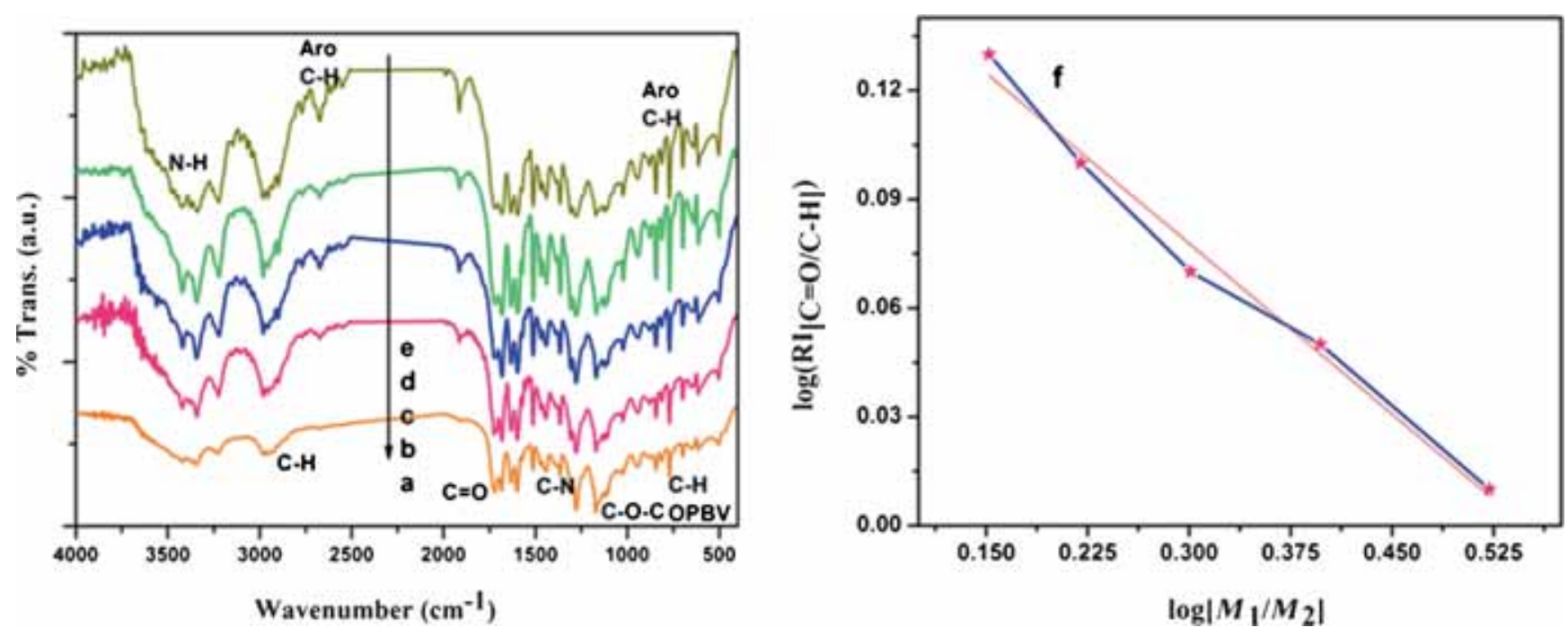

Figure 1. FTIR spectrum of poly(MEGDMA-co-4-AB) synthesised at the $\left[M_{1} / M_{2}\right]$ ratio of (a) 3.33 , (b) 2.50 , (c) 2.00 , (d) 1.66 and

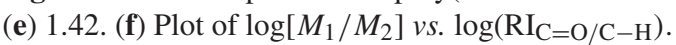


from MEGDMA units. A peak at $1726 \mathrm{~cm}^{-1}$ corresponds to the $\mathrm{C}=\mathrm{O}$ stretching of MEGDMA units. Recently, Meenarathi et al [27] reported about the FTIR spectrum of poly(caprolactone) (PCL), particularly the carbonyl stretching. The present investigation also exhibited the same. The aromatic ester carbonyl stretching of 4-AB appeared at $1681 \mathrm{~cm}^{-1}$. A peak at $1632 \mathrm{~cm}^{-1}$ indicated the presence of $\mathrm{C}=\mathrm{C}$ stretching (due to the MEGDMA). The $\mathrm{C}-\mathrm{H}$ bending vibration can be seen at $1602 \mathrm{~cm}^{-1}$. The $\mathrm{C}-\mathrm{N}$ stretching of the co-polymer is noticed at $1364 \mathrm{~cm}^{-1}$. The ester $\mathrm{C}-\mathrm{O}-\mathrm{C}$ linkage can be seen at $1161 \mathrm{~cm}^{-1}$. The $\mathrm{C}-\mathrm{H}$ out-of-plane bending vibration is noticed at $768 \mathrm{~cm}^{-1}$. The aromatic $\mathrm{C}-\mathrm{H}$ bending vibration is observed at 845 and $699 \mathrm{~cm}^{-1}$. The aromatic C-H stretching from $4-\mathrm{AB}$ can be seen at $2680 \mathrm{~cm}^{-1}$. It is very interesting to note that while increasing the $4-\mathrm{AB}$, the corrected peak area at $1681 \mathrm{~cm}^{-1}$ linearly increased. The corrected peak area of the $\mathrm{C}-\mathrm{H}$ out-of-plane bending vibration also proportionally increased. In order to find out the order of addition of aromatic primary amine with MEGDMA, the plot of $\log M_{1} / M_{2}$ vs. $\log \left(\mathrm{RI}_{\mathrm{C}=\mathrm{O} / \mathrm{C}-\mathrm{H}}\right)$, figure $1(\mathrm{f})$, was drawn and the plot showed a decreasing trend. This is due to the increase in $4-\mathrm{AB}$. The slope value was calculated as 0.31 , which confirmed the 0.31 order of reaction with respect to monomers concentration. Similar type of plot for the PCL system was reported by Kannammal and co-workers [28]. This is in accordance with the literature report. The reaction between MEGDMA and aromatic primary amine leads to AMA polymerisation. The slope value declared that, $0.31 \mathrm{~mol}$ of monomer is required to form $1 \mathrm{~mol}$ of the co-polymer.

\subsection{UV-visible reflectance study}

The above-synthesised polymer was characterised by UVvisible reflectance study. The co-polymer contains both aliphatic and aromatic ester groups with secondary or tertiary amine. Hence, ester has a chance to act as a hydrophobic segment, whereas the amine as a hydrophilic segment. Figure 2a indicates the UV-visible reflectance spectrum of $\mathrm{AB}$ type co-polymer. As a result of alternate co-polymer formation, the reflectance value was initially increased up to $650 \mathrm{~nm}$ (with $89 \%$ reflectance) corresponding to the hydrophobic nature of ester moieties. Thereafter, the reflectance value was slowly decreased due to the presence of hydrophilic amine. Recently, Kannammal et al [28] reported about the reflectance of PCL nanocomposites. When compared with the literature, the present system exhibited somewhat lower reflectance value and it is suggested that the current system has less hydrophobicity. Further studies are going on in our laboratory regarding the analysis of hydrophobic character of the poly(ester amine).

\subsection{XRD study}

The crystalline nature of the co-polymer was checked by XRD measurements. Figure $2 b$ indicates the same. The diffractogram exhibited a broad peak in the $2 \theta$ range of $15-20^{\circ}$. It is concluded that the above-synthesised polymer is an amorphous one. Generally, an amorphous polymer exhibits a broad peak in the $2 \theta$ range of $15-20^{\circ}$. Hence, it is concluded that the present system is an amorphous one. In 2009, Anbarasan et al [29] explained the XRD patterns of PVA/Mg $(\mathrm{OH})_{2}$ nanocomposite system with amorphous nature. When compared with the literature, the present system exhibits somewhat good result.

\subsection{SEM report}

The surface morphology of the co-polymer is shown in figure $2 \mathrm{c}$. The surface morphology showed that the co-polymer has a layered structure. The size of the polymer particles varied between 100 and $1000 \mathrm{~nm}$. Polymer nanoparticles formation is explained below. The combination of hydrophobic ester and hydrophilic amine leads to the formation of interface region. In this interface region, the polymer chains tend
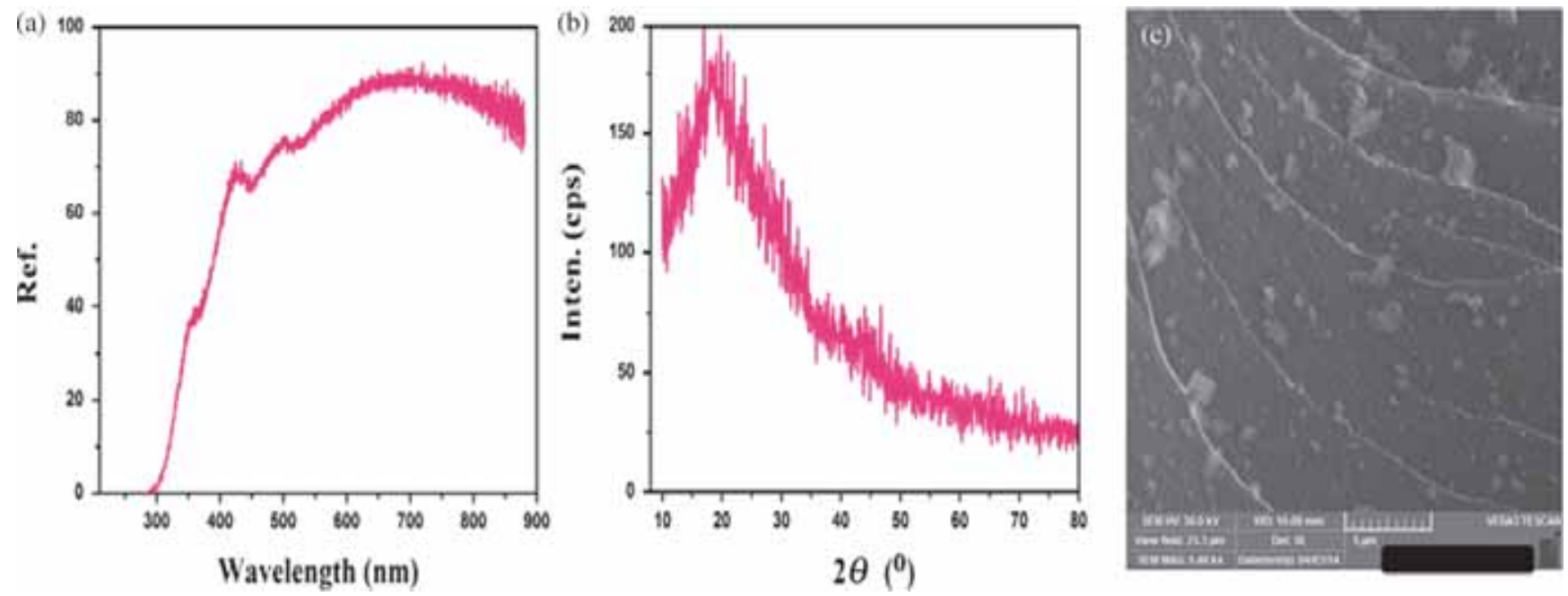

Figure 2. (a) UV-visible reflectance spectrum, (b) X-ray diffraction pattern and (c) SEM image of poly(MEGDMA-co-4-AB) synthesised at $\left[M_{1} / M_{2}\right]=2.0$. 
to align in a stereo-regular manner. This leads to the formation of polymeric nanoparticles. The polymeric nanoparticles with layered structure are a suitable candidate for the drug delivery application [30]. In the drug delivery field, the nanoparticles act as an effective drug carrier. However, the main aim of the present investigation is non-isothermal degradation kinetics of poly(ester amine) and so discussing about the drug delivery study is not quite interesting at this juncture.

\subsection{DSC analysis}

The phase transition in the polymer samples was studied by DSC. It was found that while increasing the concentration of 4-AB the first-order $T_{\mathrm{g}}$ increased (figure 3(a-e)). At lower

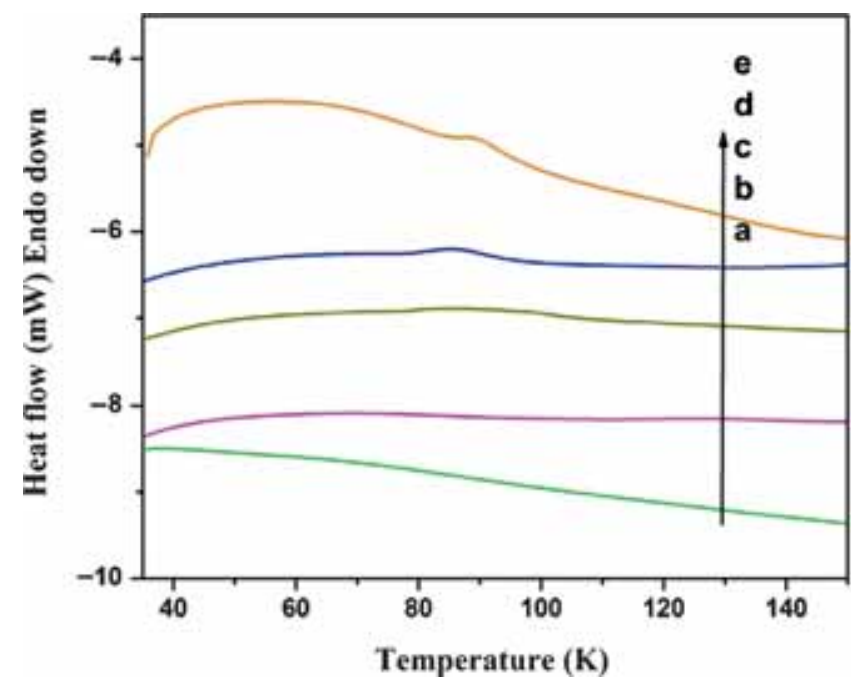

Figure 3. DSC thermogram of poly(MEGDMA-co-4-AB) synthesised at the $\left[M_{1} / M_{2}\right]$ ratio of (a) 3.33, (b) 2.50, (c) 2.00, (d) 1.66 and (e) 1.42 .

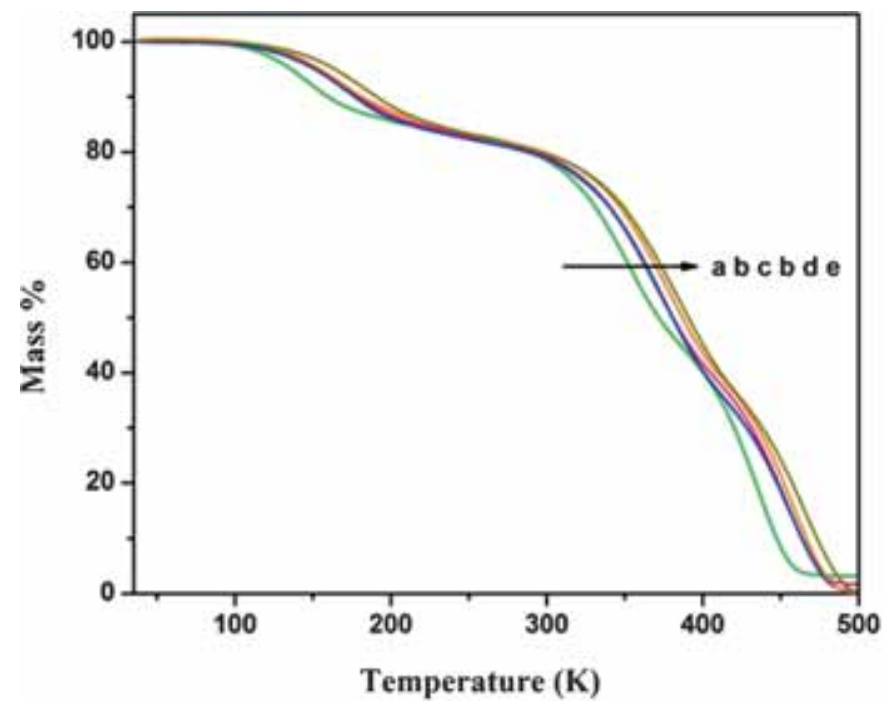

concentration of 4-AB, the first-order $T_{\mathrm{g}}$ appeared. Again it was interesting to note that a first-order $T_{\mathrm{g}}$ increased from 85.1 to $89.18^{\circ} \mathrm{C}$ and this was due to the rigid nature of $4-\mathrm{AB}$. $4-\mathrm{AB}$ contains one bulky phenyl ring and one lengthy $\mathrm{CO}_{2}$ Et group. The presence of phenyl ring leads to more rigidity, whereas the $\mathrm{CO}_{2}$-Et group leads to increase in the flexibility with steric hindrance. Due to these reasons, the first-order $T_{\mathrm{g}}$ appeared as a hump with increase in temperature. Thus, the DSC study supported the XRD report. Hence, both XRD and DSC studies jointly confirmed the amorphous nature of the co-polymer. In 2004, Wu et al [10] explained the $T_{\mathrm{g}}$ of poly(ester amine) formed by AMA polymerisation mechanism. The present system yielded an excellent result when compared with the literature report [20]. By comparison, one can come to a conclusion that the $T_{\mathrm{g}}$ of poly(ester amine) depends on the nature of the monomers used and number of repeating units being present in the polymer chain.

\subsection{TG history}

The thermal stability of the co-polymer synthesised at various concentration of $4-\mathrm{AB}$ is given in figure $4(\mathrm{a}-\mathrm{e})$. The TG thermogram exhibits a three-step degradation process. The first minor mass loss of around $175^{\circ} \mathrm{C}$ is due to the removal of unreacted monomers and physisorbed monomers. The second major mass loss of around $370^{\circ} \mathrm{C}$ is ascribed to the degradation of MEGDMA. The third major mass loss of around $460^{\circ} \mathrm{C}$ is responsible for the degradation of $\mathrm{AB}$ copolymer with the liberation of $\mathrm{CO}_{2}$. The TG results inferred that the degradation pattern of the co-polymer synthesised at five different concentrations are almost similar. At around $500^{\circ} \mathrm{C}$ all the co-polymer chains were degraded. As mentioned above, $\mathrm{Wu}$ and research team [20] reported the degradation temperature of poly(ester amine) with lower values. This can be explained on the basis of structure of the monomers, particularly MEGDMA used for the AMA polymerization reaction.

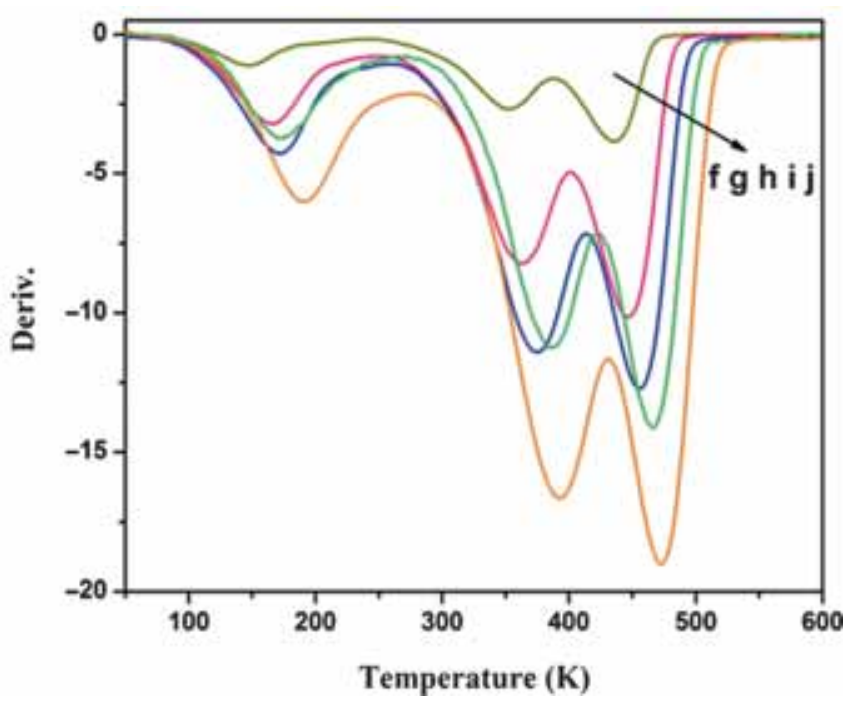

Figure 4. (a-e) TG and (f-j) DT thermogram of poly(MEGDMA-co-4-AB) synthesised at the $\left[M_{1} / M_{2}\right]$ ratio of 2.5 and at the heating rates of $(\mathbf{a}, \mathbf{f}) 5,(\mathbf{b}, \mathbf{g}) 15,(\mathbf{c}, \mathbf{h}) 20,(\mathbf{d}, \mathbf{i}) 25$ and $(\mathbf{e}, \mathbf{j}) 30^{\circ} \mathrm{C} \mathrm{\textrm {min } ^ { - 1 }}$. 
The rigid nature of 4-AB increased the $T_{\mathrm{g}}$ of the co-polymer. The deri vative curve is shown in figure $4(f-j)$. Also here one can observe that while increasing the heating rate, $T_{\mathrm{d}}$ also proportionally increased. This is due to the scanning speed.

\section{$3.7 \quad$ Non-isothermal degradation kinetics}

The degradation kinetics study of the co-polymer was used to find out the energy of activation. The present nonisothermal degradation kinetic investigation was made based on the degradation of PANI/nanocomposites [31] and poly (chontroitin-co-N-isopropylacrylamide) [32] systems. For $E_{\mathrm{a}}$ studies, the polymer sample used here is $\left[M_{1} / M_{2}\right]=2$. The polymer sample was heated at five different heating rates, such as $5,15,20,25,30^{\circ} \mathrm{C} \mathrm{min}{ }^{-1}$. Here also one can observe the TG pattern of polymer sample as mentioned above. Figure 4(a-e) indicates the TG thermogram of $\left[M_{1} / M_{2}\right]=2$. The derivative plot is shown in figure $4(\mathrm{f}-\mathrm{j})$.
It is very interesting to note that while increasing the heating rate the degradation temperature as well as the peak area were linearly increased (figure $5(\mathrm{a}-\mathrm{c})$ ).

In order to find out the energy of activation in the present investigation, three non-isothermal kinetics models were followed. The first model is FWO model (the plot of $\ln \beta v s$. $1000 / T_{\mathrm{d}}$; figure $5(\mathrm{~d}, \mathrm{~g}, \mathrm{j})$ ), Auggis-Bennet method (the plot of $\ln \left(\beta / T_{\mathrm{d}}\right) v s .1000 / T_{\mathrm{d}}$ (figure $5(\mathrm{e}, \mathrm{h}, \mathrm{k})$ ) and Kissinger method (the plot of $\ln \left(\beta / T_{\mathrm{d}}^{2}\right) v s .1000 / T_{\mathrm{d}}$ (figure $5(\mathrm{f}, \mathrm{i}, 1)$ ), for stage I, stage II and stage III, respectively, for each method. For stage I, $E_{\mathrm{a}}$ value was calculated as $156.28 \mathrm{~kJ} \mathrm{~mol}^{-1}$ by FWO model, whereas the Auggis-Bennet method and Kissinger models [33] yielded the $E_{\mathrm{a}}$ values as 135.17 and $112.20 \mathrm{~kJ} \mathrm{~mol}^{-1}$, respectively. The $E_{\mathrm{a}}$ values are within the error limit. It was found that step II consumes somewhat higher thermal energy than stage I. In overall comparison, the $E_{\text {a }}$ value calculated for three stages by Kissinger method yielded a lower one. This is due to the denominator in $Y$-axis caption.
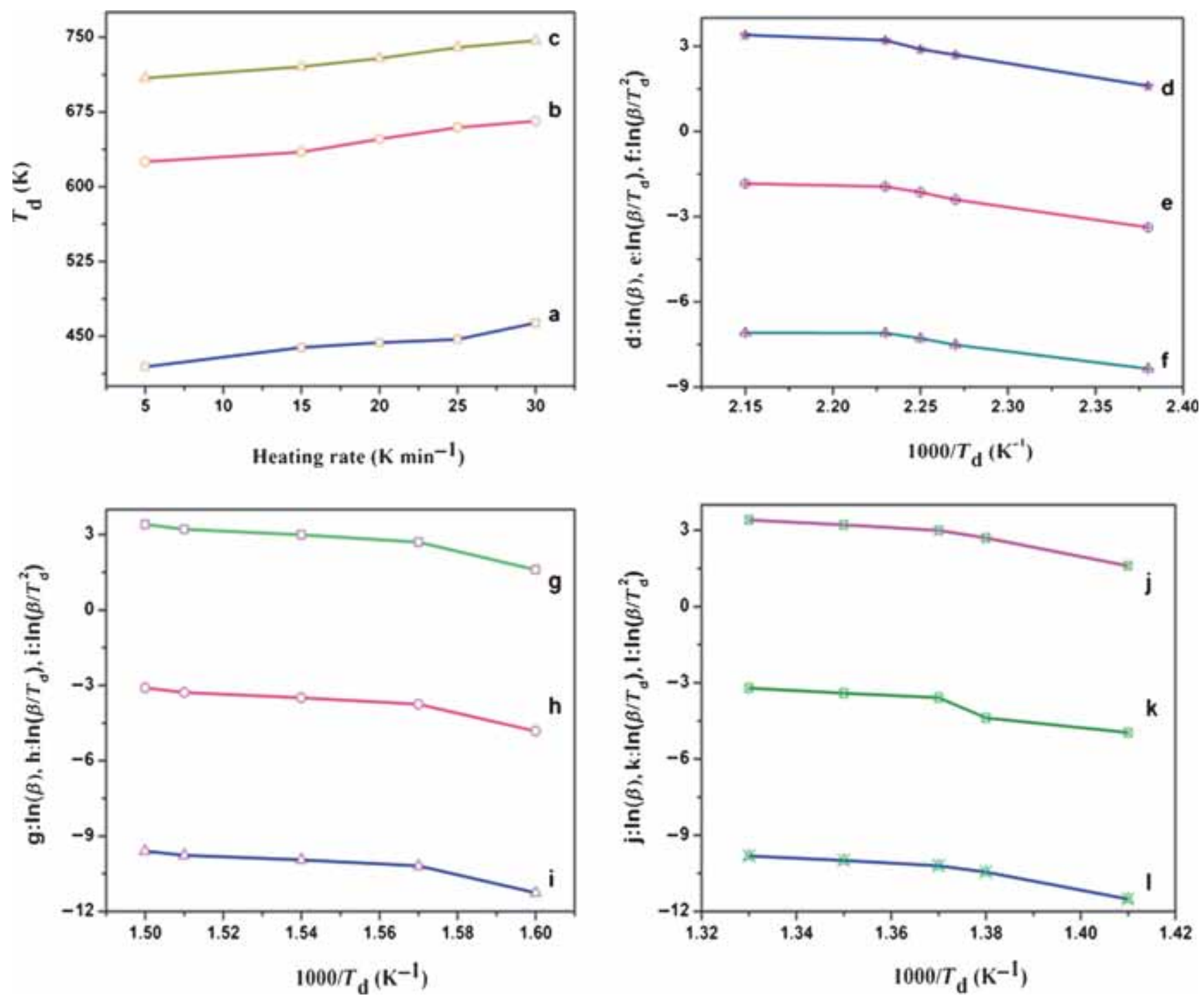

Figure 5. The plot of heating rates vs. degradation temperature: (a) stage I, (b) stage II, (c) stage III. (d, g, j) Classical plots of FlynnWall-Ozawa model, (e, h, k) Auggis-Bennet model, (f, i, l) Kissinger model for stage I, stage II and stage III, respectively. 

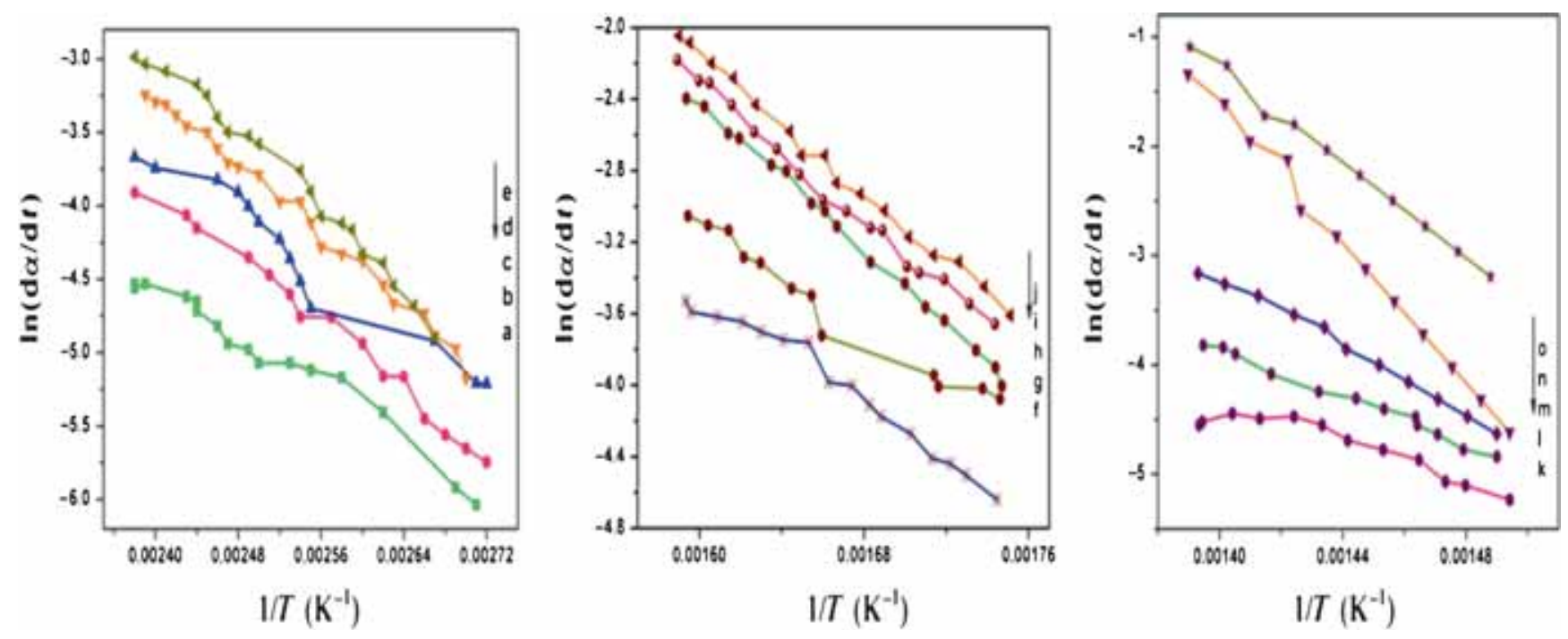

Figure 6. The Friedman plot for (a-e) stage I, (f-i) stage II and (k-o) stage III.

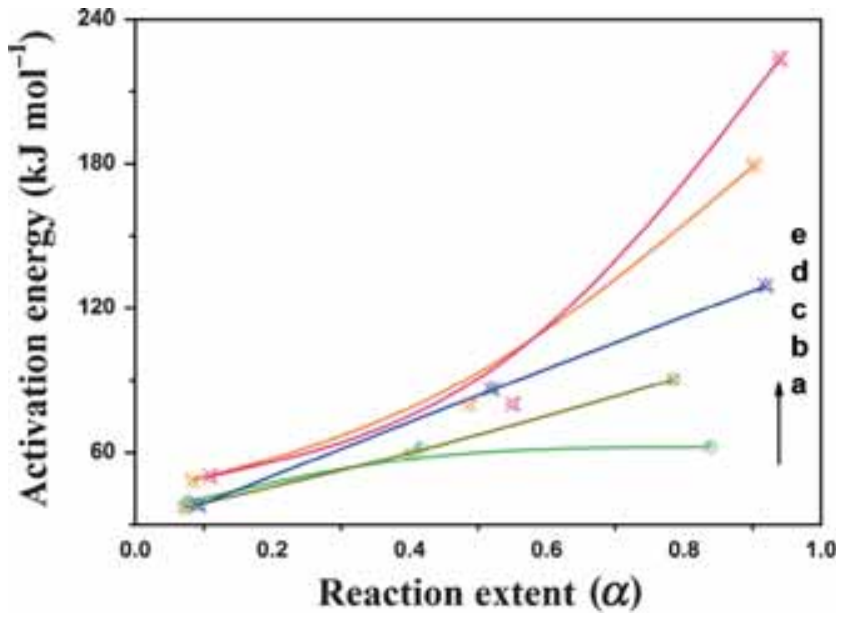

Figure 7. The plot of reaction extent against activation energy at the heating rates of (a) 5 , (b) 15 , (c) 20 , (d) 25 and (e) $30^{\circ} \mathrm{C} \mathrm{min}^{-1}$.

The activation energy was calculated for the degradation of the co-polymer by the Friedman method along with a differential method. In Friedman method, the plot of $\ln (\mathrm{d} \alpha / \mathrm{d} t)$ vs. $1 / T$ (figure $6(\mathrm{a}-\mathrm{o})$ ) was made for three stages of degradation. The plot showed a decreasing trend for all the heating rates. This is in accordance with the report of Bauri et al [22]. From the slope value, the $E_{\text {a value was calculated and plotted }}$ against the reaction extent $(\alpha)$. The plot is further discussed in figure 7 . The plot of activation energy $\left(E_{\mathrm{a}}\right)$ against reaction extent $(\alpha)$ for the co-polymer at different heating rates is given in figure $7(\mathrm{a}-\mathrm{e})$. The $E_{\mathrm{a}}$ value for the degradation of the co-polymer varied between 37.1 and $223.8 \mathrm{~kJ} \mathrm{~mol}^{-1}$. As the $E_{\text {a }}$ value was calculated at different heating rates, it is very interesting to note that, the $E_{\mathrm{a}}$ value increased gradually. Generally, it was found that, the $E_{\text {a }}$ value was gradually increased up to the $\alpha$-value of 0.5 [29-31], thereafter;

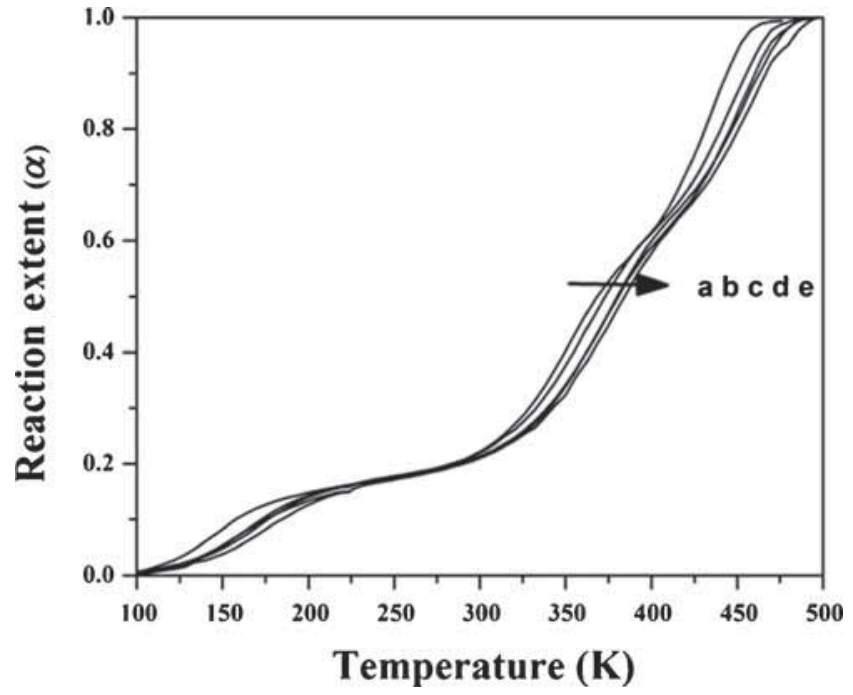

Figure 8. The plot of reaction extent against temperature at the heating rates of (a) 5 , (b) 15 , (c) 20, (d) 25 and (e) $30^{\circ} \mathrm{C} \mathrm{min}-1$ under $\mathrm{N}_{2}$ atmosphere.

it showed a sudden increase in the $E_{\mathrm{a}}$ value. It is also noted that, at lower heating rates, the $E_{\mathrm{a}}$ value linearly increased with the increase in $\alpha$-value, whereas at higher heating rates (above $25^{\circ} \mathrm{C} \mathrm{min}^{-1}$ ) the plot followed a parabolic curve. This confirmed the faster degradation rate at higher heating rates.

Before entering the kinetics of model-free methods it is necessary to know the extent of reaction conversion against temperature (figure $8(\mathrm{a}-\mathrm{e})$ ). The figure shows that, the degradation of the polymers includes the three-step degradation process. This also shows that, with increase in the heating rate the degradation temperature slightly increased. At the same time, the reaction conversion extent is decreased due to 


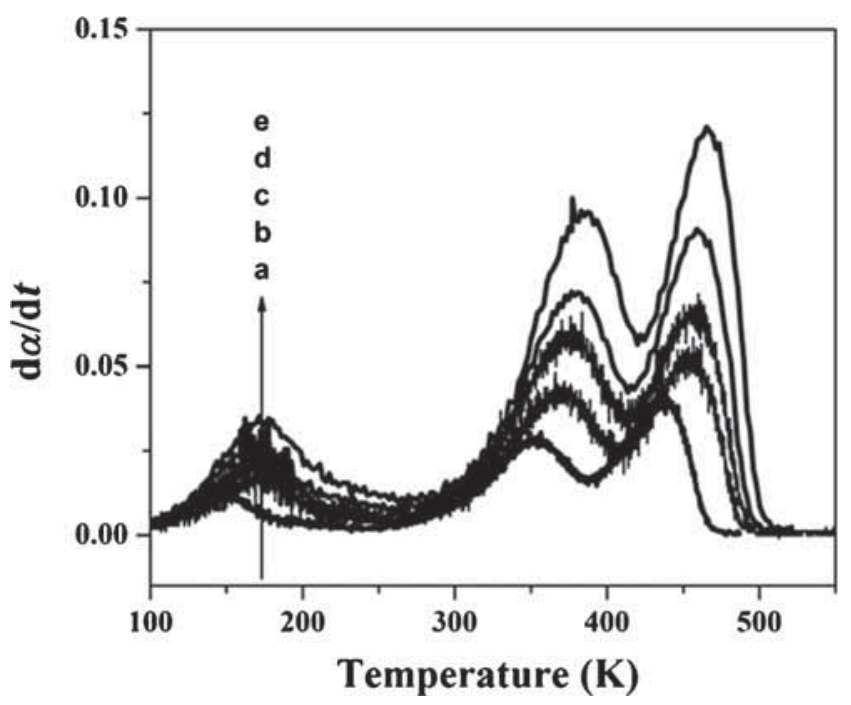

Figure 9. The plot of rate of conversion (DTG) against temperature at the heating rates of (a) 5 , (b) 15 , (c) 20 , (d) 25 and (e) $30^{\circ} \mathrm{C}$ $\min ^{-1}$ under $\mathrm{N}_{2}$ atmosphere.

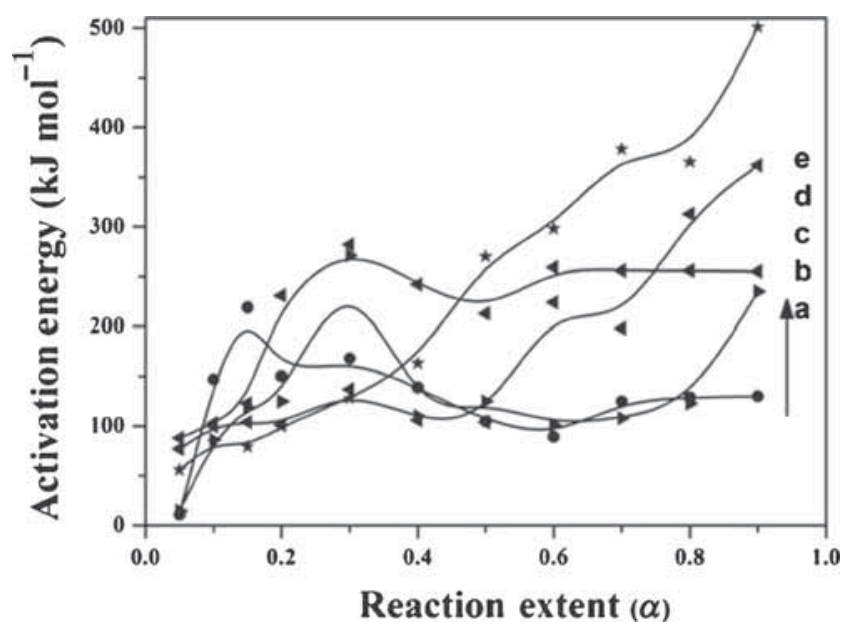

Figure 10. Dependence of apparent activation energy with the reaction extent by differential method at the heating rate of (a) 5 , (b) 15 , (c) 20 , (d) 25 and (e) $30^{\circ} \mathrm{C} \mathrm{min}^{-1}$ under $\mathrm{N}_{2}$ atmosphere.

the increase of heating rate. This is in accordance with the Goswami et al [25].

The variation of reaction extent against temperature was further confirmed by plotting $\mathrm{d} \alpha / \mathrm{d} t \quad v s$. temperature (figure $9(\mathrm{a}-\mathrm{e})$ ). Here also one can see the three-step degradation process. It is also found that, while increasing the heating rate the derivative of reaction conversion is also gradually increased with a slight increase in temperature. This is applicable to all the three-step process.

3.7a Differential isoconversional methods: The degradation kinetics of the polymer is now explained with the isoconversional differential methods. Here the degradation was carried out at $\mathrm{N}_{2}$ atmosphere. The plot of reaction extent against activation energy for the differential method is shown in figure $10(a-e)$. From the figure one can say that, for all the

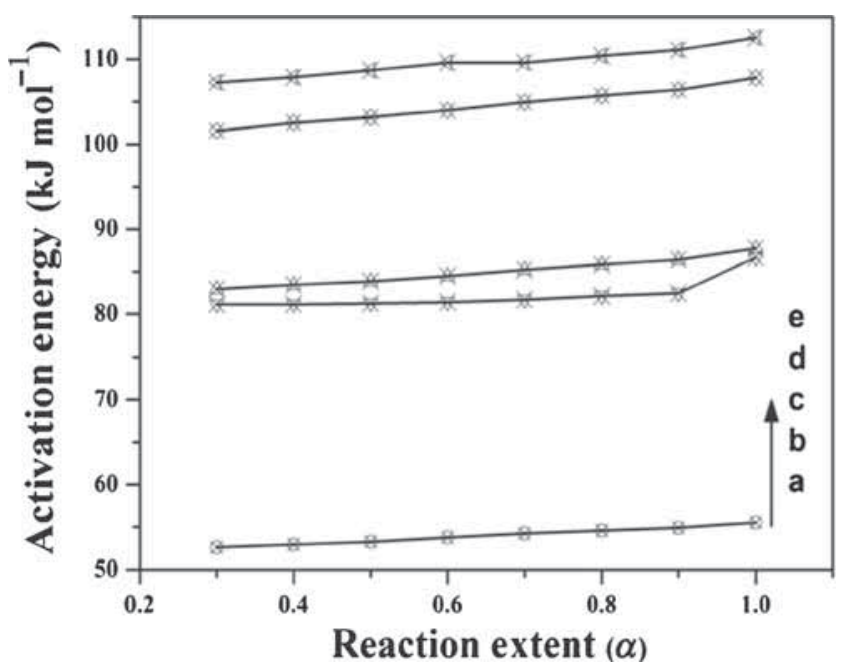

Figure 11. Dependence of apparent activation energy with the reaction extent by KAS method at the heating rate of (a) 5, (b) 15, (c) 20 , (d) 25 and (e) $30^{\circ} \mathrm{C} \mathrm{min}^{-1}$ under $\mathrm{N}_{2}$ atmosphere.

heating rates, the activation energy was found to be low during the initial stage. Beyond the $\alpha$-value 0.7 , the $E_{\mathrm{a}}$ value was drastically increased for all the heating rates.

The appearance of three reflection points at all the threestep degradation process again confirmed the three-step degradation of the polymer. When $\alpha=0.5$, the $E_{\mathrm{a}}$ value was increased to 95 and $285 \mathrm{~kJ} \mathrm{~mol}^{-1}$. At higher conversion $(\alpha=$ $0.9)$, the $E_{\mathrm{a}}$ value increased to $505 \mathrm{~kJ} \mathrm{~mol}^{-1}$.

3.7b Integral isoconversional methods: Figure 11(a-e) explains the variation of activation energy against reaction extent by KAS method. It was found that, the initial activation energies varied at different heating rates. While increasing the reaction extent the change in the activation energy is little. At $\alpha=0.5$, the $E_{\mathrm{a}}$ value varies between 52 and $108 \mathrm{~kJ} \mathrm{~mol}^{-1}$. At the maximum $\alpha$-value, the $E_{\mathrm{a}}$ value varies between 55 and $112 \mathrm{~kJ} \mathrm{~mol}^{-1}$. Recently, Goswami et al [25] explained that the increase in activation energy was attributed to the rate-limiting step of random chain session. The results and discussion of the present investigation coincide with Goswami et al publications.

\section{Conclusion}

The niche points are presented here as conclusion. The peak at $1683 \mathrm{~cm}^{-1}$ in the FTIR spectroscopy was gradually increased with the increase in concentration of 4-AB. The FTIR-RI study indicated the 0.30 order of reaction with respect to monomers concentration. The UV-visible reflectance spectrum suggested the formation of amphiphilic-like polymer. The XRD and DSC studies jointly declared the amorphous nature of the polymer. While increasing the [4-AB], the thermal stability of the co-polymer slightly increased, due to the rigid nature of phenyl ring present in 
the 4-AB. The Kissinger method yielded the lowest $E_{\text {a }}$ for the degradation kinetics of the co-polymer. By increasing the heating rate the $T_{\mathrm{d}}$ also increased gradually. The Friedman method indicated that the $E_{\mathrm{a}}$ value for the degradation of the co-polymer varied between 37.1 and $223.8 \mathrm{~kJ} \mathrm{~mol}^{-1}$. SEM morphology analyses indicated the formation of layered structure with the simultaneous formation of 100-1000 nm sized polymeric nanoparticles. The present methodology is a solvent- and catalyst-free method.

\section{Acknowledgement}

Dr G John David Raja, Assistant Professor, Department of English, Thiagarajar College, Madurai, is gratefully acknowledged for his valuable help during this manuscript preparation.

\section{References}

[1] Yang J M, Ji S J, Gu D G, Shen Z L and Wang S Y 2005 J. Organomet. Chem. 6902989

[2] Tamami B, Fadavi A and Tamami M 2006 Iran Polym. J. 15 789

[3] Roy A, Kundu D, Kundu S K, Majee A and Hajra A 2010 Open Catal. J. 334

[4] Escalante J, Morales M C and Linzaga I 2008 Molecules 13 340

[5] Kou J P, Lu Y J, Luo X Y and Li J Z 2009 Chem. Res. Chin. Univ. 25461

[6] Li G Z, Randev R K, Rees G, Boyer C, Tong Z and Haddleton K 2010 Polym. Chem. 11196

[7] Movassagha B and Shaygana P 2006 Arkivoc 12130

[8] Reddick J J, Cheng J and Roush W R 2003 Org. Lett. 51967

[9] Sarathi P A, Gnanasekaran C and Shanmugasundaram A 2008 Bull. Korean Chem. Soc. 29790

[10] Mukherjee C and Misra A K 2007 Lett. Org. Chem. 454

[11] Bosica G, Spiteri J and Borg C 2014 Tetrahedron 702449

[12] Espeel P, Goethals F, Driessen F, Nguyen L T H and Prez F E D 2013 Polym. Chem. 92449
[13] Mather B D, Viswanathan K, Millar K M and Long T E 2006 Prog. Polym. Sci. 31487

[14] Ying A G, Wang L M, Deng H X, Chen J H, Chen X Z and Ye W D 2009 Arkivoc 11288

[15] Kim T I, Seo H J, Baek J H, Park J H and Park J S 2005 Bull. Korean Chem. Soc. 26175

[16] Tsai P F, Chang W Y, Hsiao Y C, Li K J and Shau M D 2012 Molecules 179056

[17] Brey D M, Ifkovits J L, Mozia R I, Katz J S and Burdick J A 2008 Acta Biomater. 11207

[18] Gharat V D and Shertukade U V 2014 Intern. J. Sci. Res. Pub. 111

[19] Bhise N S, Gray R S, Sunshine J C, Htet S, Ewald A J and Green J J 2010 Biomaterials 318088

[20] Wu D, Liu Y, He C, Chung V and Goh S 2004 Macromolecules 376763

[21] Zugates G T, Tedford N C, Zumbuehl A, Kang C S, Griffith L G, Langer R and Anderson D G 2007 Bioconj. Chem. 18 1887

[22] Bauri K, Roy S G, Arora S, Dey R K, Goswami A, Madras G and De P 2013 J. Therm. Anal. Calorim. 111753

[23] Goswami S and Kiren K 2012 Bull. Mater. Sci. 35657

[24] Vyazovkin S V 2001 J. Comput. Chem. 22178

[25] Goswami A, Srivastava G, Umarji A M and Madras G 2012 Thermochim. Acta 54753

[26] Doyle C D 1961 J. Appl. Polym. Sci. 5285

[27] Meenarathi B, Palanikumar S, Kannammal L and Anbarasan R 2015 Spectrochim. Acta Part A 13593

[28] Kannammal L, Palanikumar S, Meenarathi B, Yelilarasi A and Anbarasan R 2014 J. Phys. D Appl. Phys. 471

[29] Anbarasan R, Barsanabanu B, Inigo M and Dhanalakshmi V 2009 Int. J. Nanosci. 8381

[30] Kailash S, Meenarathi B, Palanikumar S and Anbarasan R 2015 Int. J. Polym. Mater. Polym. Biomater. 64620

[31] Zhang S, Wang S, Huang S, Li Y and Tan Z 2015 J. Therm. Anal. Calorim. 1191853

[32] Costa Sanches S C, Vasconcelos F, Marinho P and Costa R 2015 J. Thermal. Anal. Calorim. 120991

[33] Vincent B J and Natarajan B 2015 J. Therm. Anal. Calorim. 1191373 